Jul 1st, 12:00 AM

\title{
Simple Spatially Distributed Model of the Global Carbon Cycle and its Dynamic Properties
}

Yuri Svirezhev

Follow this and additional works at: https://scholarsarchive.byu.edu/iemssconference

Svirezhev, Yuri, "Simple Spatially Distributed Model of the Global Carbon Cycle and its Dynamic Properties" (2002). International Congress on Environmental Modelling and Software. 94.

https://scholarsarchive.byu.edu/iemssconference/2002/all/94

This Event is brought to you for free and open access by the Civil and Environmental Engineering at BYU ScholarsArchive. It has been accepted for inclusion in International Congress on Environmental Modelling and Software by an authorized administrator of BYU ScholarsArchive. For more information, please contact scholarsarchive@byu.edu, ellen_amatangelo@byu.edu. 


\title{
Simple Spatially Distributed Model of the Global Carbon Cycle and its Dynamic Properties
}

\author{
$\underline{\text { Yuri Svirezhev }}$ \\ Potsdam Institute for Climate Impact Research, Potsdam \\ P.O. Box 601203 D-14412 Potsdam, Germany \\ E-mail: Yuri.Svirezhev@pik-potsdam.de
}

\begin{abstract}
A specially simplified spatially distributed model of the Global Carbon Cycle (GCC) is presented that is allowed to get a general solution and to study its basic dynamic properties (like spectral portrait, composition and decomposition of solutions, etc.). Assuming that the productivity depends linearly on concentration of carbon in the atmosphere, the problem is reduced to a standard linear one. For calibration the Bazilevich data containing information on plant productivity, living biomass and soil organic matter of different biomes is used. Calculating the matrix eigenvalues of the basic linear problem we get its spectral portrait. The characteristic times change sufficiently smoothly from several years to several hundreds. Among all the exponentially damped solutions there are three oscillating ones with very long periods: from 100 to 4,000 years. This portrait is very stable in relation to variations in the areas, covered by different biomes. Therefore, the terrestrial part of the GCC is a well-structured dynamic system with very wide opportunities to compensate disturbances with different time characteristics.

Note that any distribution is described by its statistical moments and namely these moments are very robust in respect to numerical meanings of the distributed value. A special operation of convolution to the general solution of a spatially distributed model is suggested. This allows getting a dependence of convoluted solution on first moments such as means and covariance. One can see that non-homogeneity of these distributions on the whole increases the amount of carbon in the atmosphere.
\end{abstract}

Key words: Global Carbon Cycle; Modelling.

\section{INTRODUCTION}

There are a lot of different models of the Global Carbon Cycle (GCC). They differ from each other by their complexity and the degree of detail, but they possess some common dynamical properties (assuming they more and less adequately reflect reality). The "zoom" of consideration allows picking out two scales of modelling. The first scale is when the whole planet is considered consisting of a relatively small number of compartments (atmosphere, terrestrial biota, soils, ocean and oceanic biota). The second is, when, for instance, the terrestrial biota consists of different types of vegetation, and soils are also distributed on the Globe in accordance with some typology. The problem is to select the most typical representatives from these immense sets.

Typical models, which belong to the first set, are the models of Kwon and Schnoor [1994] and Svirezhev et al. [1999]. They are sufficiently simple and their dynamic properties can be studied analytically. The second pole is occupied by models, which include both physiological and biochemical processes determining the productivity of different plants and also a spatial distribution of environmental conditions, in which all these processes are functioning and are described in detail. For these models no analytical solutions are presently available. Examples of this type of models are HYBRID V3.0 (Friend et al. [1997]), BIOME3 (Haxeltine and Prentice, [1996]), and the model of Foley et al. [1996].

Any distribution is described by its statistical moments and namely the moments are very robust in respect to numerical meanings of the distributed value. This suggests applying a special convolution operation to the general solution of a spatially distributed model in order to get a dependence of the convoluted solution on the first moments such as means and covariance.

\section{MODEL}


Let us consider the terrestrial part of the Global Carbon Cycle (GCC) as a partially isolated dynamical system. We assume that the carbon in the atmosphere is intermixed entirely during $3-4$ months, therefore, if a model step of one year (and more) is used then the atmosphere can be considered as a single compartment.

We neglect also all horizontal flows of the terrestrial carbon in comparison with the vertical ones. Then the simplest form for the spatially distributed model of the GCC will be:

$$
\begin{gathered}
\frac{\partial B}{\partial t}=P(C, B, x, y)-m(x, y) B, \\
\frac{\partial D}{\partial t}=m(x, y) B-\delta(x, y) D, \\
\frac{d C(t)}{d t}=-\int_{\Omega} P d \omega+\int_{\Omega} \delta D d \omega+ \\
\quad+\int_{\Omega} q(x, y, t) d \omega-Q_{o c}, \\
d \omega=d x d y, \\
\frac{d C_{o c}(t)}{d t}=Q_{o c}
\end{gathered}
$$

where $C(t)$ and $C_{o c}(t)$ are the total amounts carbon in the atmosphere and ocean, respectively. $B(x, y, t)$ is the density of living biomass at point $(x, y)$ (in carbon units), $D(x, y, t)$ is the same for the dead organic matter (humus), $m(x, y)=1 / \tau_{B}, \delta(x, y)=1 / \tau_{D}$, where $\tau_{B}(x, y)$ and $\tau_{D}(x, y)$ are residence times for carbon in living and dead biomass, respectively. Therefore, the values $m$ and $\delta$ are inverse residence times. The flow $q(x, y, t)$ is the local anthropogenic emission of carbon, the flow $Q_{o c}$ describes an exchange between the atmosphere and the ocean. The function $P=P(C, B, x, y)$ is the annual net primary production (or, productivity) at some given point of the Earth's surface. The integration is made on all surface $(\Omega)$ covered by vegetation.

System (1)-(4) has the following integral reflecting the mass conservation law:

$$
C(t)=A_{0}+\int_{0 \Omega}^{t} \int_{0} q d \omega d t-\int_{\Omega} B d \omega-\int_{\Omega} D d \omega-C_{o c}(t),
$$

where $A_{0}=C_{0}+\int_{\Omega_{0}} B_{0} d \omega+\int_{\Omega_{0}} D_{0} d \omega+\left(C_{o c}\right)_{0}$ is the total amount of carbon in the biosphere at some initial moment of time and $\Omega_{0}$ is the territory covered by the vegetation at the same time. In this expression the two items, $\left(C_{o c}\right)_{0}$ and $C_{o c}(t)$, are much larger (by one and a half orders of magnitude) than the others. The asymptotic analysis is shown that if the modelling time is by order of one hundred years then the basic system can be fibered onto two separate subsystems, each of which describes the terrestrial and oceanic parts of the GCC. For this we have to reduce the total flow of anthropogenic emission by some constant factor.

Really the productivity and the decomposition rate do not depend directly on geographical coordinates at a given point, they depend on local climatic conditions, in particular, on temperature and precipitation. Therefore, if we want to estimate the GCC reaction to climate change, we must know the explicit dependencies of these values (productivity and decomposition) on climatic parameters. We assume that $P=\alpha(T, H) C(t)$, i.e. the production $P$ is a linear function of $C$, which depends also on the annual local temperature $T(x, y)$ and precipitation $H(x, y)$. By the same token, $P$ depends implicitly on spatial co-ordinates $x$ and $y$. We also assume that $P$ does not depend on $B$. This is correct if $B$ is far from zero.

As to the linear dependence of $P$ on $C$ then this can explain the fact that the contemporary $\mathrm{CO}_{2}$ concentration in the atmosphere is approximately equal to half the saturation concentration for photosynthesis $(750-800 \mathrm{ppm})$. Therefore, the linear dependence is an entirely satisfactory approximation at the vicinity of the contemporary state. Note that we shall use our model only till $\mathrm{CO}_{2}$-doubling values.

Taking into account all these arguments the system (1)-(4) is re-written in the following linear form:

$$
\begin{aligned}
\frac{\partial B}{\partial t}=\alpha(x, y)\left(A-\int_{\Omega} B d \omega-\int_{\Omega} D d \omega\right)- \\
\quad-m(x, y) B \\
\frac{\partial D}{\partial t}=m(x, y) B-\delta(x, y) D,
\end{aligned}
$$

where the value

$$
\begin{aligned}
& A(\varepsilon t)=C(t)+\int_{\Omega} B d \omega+\int_{\Omega} D d \omega= \\
& =C_{0}+\int_{\Omega_{0}} B_{0} d \omega+\int_{\Omega_{0}} D_{0} d \omega+\int_{0}^{t} \int_{\Omega} q d \omega d t
\end{aligned}
$$

changes in respect to slow time $\varepsilon t$, i.e. the value $A$ can be considered a constant in respect to normal time $t$.

\section{CALIBRATION: METHOD AND MATERIAL}

In order to calibrate the model we must make one very important assumption: the contemporary state of the biosphere is stationary (with respect to the GCC). In this case, if the contemporary spatial distributions of the production, living biomass and dead organic matter (humus) are known then we can calculate their spatial distributions for functions $m, \delta$ and $\alpha$. Under the conditions $\partial B / \partial t=\partial D / \partial t=0$ from (6)-(7) we get 


$$
\begin{aligned}
& m(x, y)=\frac{P^{*}(x, y)}{B^{*}(x, y)}, \delta(x, y)=\frac{P^{*}(x, y)}{D^{*}(x, y)}, \\
& \alpha(x, y)=\frac{P^{*}(x, y)}{C^{*}},
\end{aligned}
$$

where the asterisk indicates belonging to a stationary state. The problem is how to construct the distributions for $P^{*}, B^{*}$ and $D^{*}$, if the real data is presented in the form of mean values for different types of vegetation (biomes)?

We used Bazilevich's data [1969, 1979, 1986, 1993] applying the special procedure of smoothing and correction (Svirezhev et al. [1985]). For the correction we also used the data of the radiocarbon storage collected by Cherkinsky (Cherkinsky, Brovkin, [1991]).

Such a type of the data organisation forces us to change the basic model, transforming it into a discrete form. Let $S_{i}(i=1, \ldots, n)$ be area of the territory occupied by the $i$-th type of vegetation (biome). We considered 28 spatial cells contained by 28 different types of vegetation, so that $n=28$. We assume that all the cells cover the whole of the terrestrial land. If $B_{i}$ and $D_{i}$ are the mean densities of living biomass and dead organic matter for $i$-th biome then the discrete form of the basic continuous model (6)-(7) is presented as

$$
\begin{aligned}
& \frac{d B_{i}}{d t}=\alpha_{i}\left(A-\sum_{j} B_{j} S_{j}-\sum_{j} D_{j} S_{j}\right)-m_{i} B_{i}, \\
& \frac{d D_{i}}{d t}=m_{i} B_{i}-\delta_{i} D_{i}, i=1, \ldots, 28 \\
& A=A_{0}+\int_{0}^{t} Q(\tau) d \tau,
\end{aligned}
$$

where $Q(t)=\int_{\Omega} q(x, y, t) d \omega$. We assume that the

biome areas change very slowly in comparison with changes of living and dead organics, i.e.

$d \ln B_{i} / d t, d \ln D_{i} / d t>>d \ln S_{i} / d t$.

Using (8) we can calculate all the coefficients of (9)-(11):

$$
\begin{aligned}
& \quad \alpha_{i}=P_{i}^{*} / C^{*}, m_{i}=\frac{1}{\tau_{B}^{i}}=P_{i}^{*} / B_{i}^{*}, \\
& \delta_{i}=\frac{1}{\tau_{D}^{i}}=P_{i}^{*} / D_{i}^{*}
\end{aligned}
$$

where $\tau_{B}^{i}$ and $\tau_{D}^{i}$ are residence times for carbon in living and dead biomass of the $i$-th biome.

\section{A SPECTRAL PORTRAIT OF THE SYSTEM}

Introducing matrix and vector notations

$$
\begin{aligned}
& \mathbf{x}=\left\{B_{1}, \ldots, B_{n}, D_{1}, \ldots, D_{n}\right\}, \\
& \mathbf{f}=\left\{\alpha_{1} A, \ldots, \alpha_{n} A, 0, \ldots, 0\right\}, \mathbf{F}=\left\|\begin{array}{l}
\mathbf{F}^{11} \mathbf{F}^{12} \\
\mathbf{F}^{21} \mathbf{F}^{22}
\end{array}\right\|
\end{aligned}
$$

where

$$
\begin{aligned}
& \mathbf{F}^{11}=\left\{-\alpha_{i} S_{j}-m_{i} \Delta_{i}^{j}\right\}, \mathbf{F}^{12}=\left\{-\alpha_{i} S_{j}\right\}, \\
& \mathbf{F}^{21}=\left\{m_{i} \Delta_{i}^{j}\right\}, \mathbf{F}^{22}=\left\{-\delta_{i} \Delta_{i}^{j}\right\}, i, j=1, \ldots, n
\end{aligned}
$$

and

$$
\Delta_{j}^{i}=\left\{\begin{array}{ll}
1, & \text { if } i=j \\
0, & \text { if } i \neq j
\end{array}\right. \text { is the Kronecker symbol, }
$$

the system (9)-(11) is written in a standard matrix form:

$$
\frac{d \mathbf{x}}{d t}=\mathbf{F x}+\mathbf{f} .
$$

By calculating the eigenvalues $\lambda_{i}, i=1, \ldots, 2 n$ of the matrix $\mathbf{F}$ we have information about dynamic properties for such a system as the GCC. All the real parts of them are negative, i.e. the single equilibrium of the system is stable. The values $\theta_{i}=1 /\left|\lambda_{i}\right|$ can be considered as characteristic times of the system. By ordering $\theta_{i}$ in ascending order we draw a graph of the dependence of $\theta_{i}$ on its position. The graph is called a spectral portrait of the system (Fig. 1).

There are three pairs of complex conjugate eigenvalues among them:

$\lambda_{a b}=-0.124 \pm 0.054 i, \lambda_{c d}=-0.057 \pm 0.0015 i$

and $\lambda_{e f}=-0.034 \pm 0.0027 i$. This indicates that oscillations with the periods $T_{a b}=2 \pi / 0.054 \approx 116$ years, $T_{c d}=2 \pi / 0.0015 \approx 4186$ years and $T_{e f}=$ $2 \pi / 0.0027 \approx 2326$ years can exist in the system. All these oscillations are rapidly damping (in the course of 8, 18 and 29 years).

This spectral portrait is very stable in relation to the variations of areas covered by different types of vegetation. Therefore, the terrestrial part of the GCC is a well-structured dynamic system with very wide opportunities to compensate any disturbances with different time characteristics. 


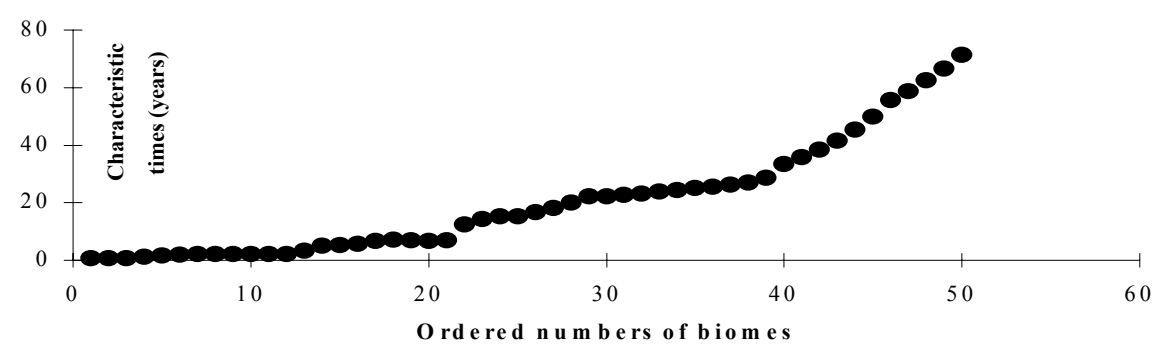

Figure 1. Spectral portrait of the terrestrial part of the GCC. This is a graph of $\theta_{i}=1 /\left|\lambda_{i}\right|$ ordered in ascending order.

\section{ATMOSPHERE CARBON DYNAMICS AS A FUNCTION OF STATISTICAL MOMENTS OF $\pi, m, \delta$ DISTRIBUTIONS}

Using the variable transformation

$u(\omega)=\left(B-B^{*}\right) / B^{*}, v(\omega)=\left(D-D^{*}\right) / D^{*}$,

system (9)-(11) is presented in the following continuous form $(\omega=(x, y))$ :

$$
\frac{\partial u}{\partial t}=m(\omega)(z-u), \frac{\partial v}{\partial t}=\delta(\omega)(u-v),
$$

where

$$
\begin{aligned}
& z(t)=\left(C-C^{*}\right) / C^{*}= \\
& =\int_{0}^{t} \frac{Q(\tau)}{C^{*}} d \tau-\frac{1}{S} \int_{\Omega}\left(\frac{\pi(\omega)}{m(\omega)} u+\frac{\pi(\omega)}{\delta(\omega)} v\right) d \omega,
\end{aligned}
$$

$\pi(\omega)=P^{*}(\omega) S / C^{*}$ and $S$ is the total area covered by vegetation. Using these dependencies the equation for $z(t)$ is presented as

$$
\frac{d z}{d t}+\hat{\pi} z=\frac{Q(t)}{C^{*}}+\frac{1}{S} \int_{\Omega} \pi v d \omega, \hat{\pi}=\frac{1}{S} \int_{\Omega} \pi d \omega .
$$

Considering this equation and equation (15) as linear in respect to $z, u$ and $v$ under the initial conditions $z(0)=u(\omega, 0)=v(\omega, 0)=0$ we get

$$
\begin{aligned}
& z(t)=F(t)+ \\
& +\frac{1}{S} \int_{0}^{t} \exp [-\hat{\pi}(t-\tau)] \int_{\Omega}^{\tau} \int_{0}^{\tau} \int_{0}^{\xi} \Phi(\omega, \tau, \xi, \eta) z(\eta) d \omega d \tau d \xi d \eta
\end{aligned}
$$

where

$$
\begin{gathered}
\Phi(\omega, \tau, \xi, \eta)=\pi(\omega) \delta(\omega) m(\omega) \cdot \\
\cdot \exp [-\delta(\omega)(\tau-\xi)] \exp [-m(\omega)(\xi-\eta)]
\end{gathered}
$$

and $F(t)=\int_{0}^{t} \frac{Q(\tau)}{C^{*}} \exp [-\hat{t}(t-\tau)] d \tau$. It is obvious that the solution of this integral equation depends on the statistical characteristics of the spatial distributions $\pi(\omega), \delta(\omega)$ and $m(\omega)$. At the first step we can restrict only the first and second moments: the means $\hat{\pi}, \hat{\delta}, \hat{m}$ and the elements of covariance matrix $\sigma_{i j}^{2}, i, j=1,2,3$ where the indices $i, j$ take the values $\pi, m, \delta$. Then

$$
z(t) \approx \hat{z}(t)+\sum_{i, j=1}^{3} \sigma_{i j}^{2} f_{i j}(t)
$$

where the term $z(t)$ depends only on the means $\hat{\pi}, \hat{\delta}, \hat{m}$. The functions $f_{i j}$ are so-called functions of influence for corresponding variance or covariance. In other words, they describe a degree of dependence of the total atmosphere carbon on the spatial diversity of soils and vegetation and spatial correlation between them. The method of construction of the solution $z(t)$ is described in Appendix.

\section{ASYMPTOTIC SOLUTION FOR ATMOSPHERE CARBON}

Let $C^{*}=750 \mathrm{Gt}$. The values of means and the elements of the covariance matrix are equal to $\hat{\pi}=7.65 \cdot 10^{-2}, \hat{\delta}=4.63 \cdot 10^{-2}, \hat{m}=22.8 \cdot 10^{-2}$,

$\sigma_{\pi \pi}^{2}=3.85 \cdot 10^{-3}, \sigma_{\pi \delta}^{2}=9.12 \cdot 10^{-4}$,

$\sigma_{\pi m}^{2}=-6.3 \cdot 10^{-3}$,

$\sigma_{\delta \delta}^{2}=4.39 \cdot 10^{-4}$,

$\sigma_{\delta m}^{2}=-1.1 \cdot 10^{-3}$ and $\sigma_{m m}^{2}=2.92 \cdot 10^{-2}$.

It is better to operate with normalised values such as variation and correlation coefficients:

$$
\begin{aligned}
& w_{\pi}=\sigma_{\pi \pi} / \hat{\pi}, w_{m}=\sigma_{m m} / \hat{m}, w_{\delta}=\sigma_{\delta \delta} / \hat{\delta}, \\
& \rho_{\delta m}=\sigma_{\delta m}^{2} / \sigma_{\delta \delta} \sigma_{m m}, \rho_{\pi m}=\sigma_{\pi m}^{2} / \sigma_{\pi \pi} \sigma_{m m}, \\
& \rho_{\pi \delta}=\sigma_{\pi \delta}^{2} / \sigma_{\pi \pi} \sigma_{\delta \delta} .
\end{aligned}
$$


Recalculating the covariance values we get: $w_{\pi}=0.81, w_{m}=0.75, w_{\delta}=0.45, \rho_{\delta m}=-0.31$, $\rho_{\pi n}=-0.6, \rho_{\pi \delta}=0.7$. The expression (19) for general solution can be re-written as

$$
z(t) \approx \hat{z}(t)+\sum_{i, j=1}^{3} \rho_{i j} w_{i} w_{j} \bar{f}_{i j}(t)
$$

where $\rho_{i i}=1$ and $\bar{f}_{i j}=\hat{x}_{i} \hat{x}_{j} f_{i j} ; \hat{x}_{i}=\hat{\pi}, \hat{m}, \hat{\delta}$. We also get such global characteristics as global productivity $P^{*}=57.4 \mathrm{Gt} /$ year, global biomass $B^{*}$ $=725 \mathrm{Gt}$ and global dead organic matter $D^{*}=$ $1285 \mathrm{Gt}$ of carbon. Therefore, the solution $\hat{z}(t)$ is written as

$\hat{z}(t)=\int_{0}^{t} \frac{Q(t-\tau)}{C^{*}}\left\{\begin{array}{l}0.335+ \\ +5.8 \exp (-0.175 \tau) \sin (0.027 \tau)\end{array}\right\} d \tau$

We assume that the dynamics of emission is described by Bolin's BAU-scenario: $Q(t)=3.5 \exp (0.029) \mathrm{Gt} /$ year . Calculating the function of influence $\bar{f}_{i j}$ and summing all these influences with the values of variation and correlation coefficients we get from (20) the more realistic "exact" solution $z(t)$. The nonhomogeneity of the distributions $\pi, m$ and $\delta$ increases the amount of carbon in the atmosphere. If comparing our prognosis with the others, using more "complex" and "geographically explicit" models, then the difference is minor. For instance, the "exact" solution is practically indistinguishable from so-called a "well-balanced" prognosis by den Elzen, Beusen and Rotmans [1995].

\section{CONCLUSIONS}

The simplifications, which do not principally distort the main mechanisms of the GCC, allowed us to gain a lot of information about many properties of this dynamical system. Its spectral portrait shows that there are neither specific characteristic times nor a fibering of phase space, which could allow to construct a correct asymptotics: characteristic times change sufficiently smooth from several years to several hundreds. Among all the exponentially damped solutions there are three oscillating ones with very long periods.

\section{APPENDIX. CONSTRUCTION OF THE SOLUTION $Z(t)$}

If the distributions $\pi(\omega), \delta(\omega)$ and $m(\omega)$ are given then the means and the elements of the covariance matrix are calculated as
$\hat{\pi}=\frac{1}{S} \int_{\Omega} \pi d \omega, \quad \hat{\delta}=\frac{1}{S} \int_{\Omega} \delta d \omega, \hat{m}=\frac{1}{S} \int_{\Omega} m d \omega \quad$ and

$\sigma_{\pi \pi}^{2}=\frac{1}{S} \int_{\Omega}(\Delta \pi)^{2} d \omega, \quad \sigma_{\pi \delta}^{2}=\frac{1}{S} \int_{\Omega} \Delta \pi \Delta \delta d \omega$,

$\sigma_{\pi m}^{2}=\frac{1}{S} \int_{\Omega} \Delta \pi \Delta m d \omega, \quad \sigma_{\delta \delta}^{2}=\frac{1}{S} \int_{\Omega}(\Delta \delta)^{2} d \omega$,

$\sigma_{\pi m}^{2}=\frac{1}{S} \int_{\Omega} \Delta \delta \Delta m d \omega, \quad \sigma_{m m}^{2}=\frac{1}{S} \int_{\Omega}(\Delta m)^{2} d \omega$,

where $\Delta \pi=\pi-\hat{\pi}, \Delta \delta=\delta-\hat{\delta}, \Delta m=m-\hat{m}$.

We construct the solution $z(t)$ in the form of the following expansion:

$z(t) \approx \hat{z}(t)+\sum_{i, j=1}^{3} \sigma_{i j}^{2} f_{i j}(t)$

Let the function $\Phi(\omega, \tau, \xi, \eta)$ also be represented in the form of the expansion:

$\Phi(\pi, \delta, m ; \tau, \xi, \eta) \approx \Phi(\hat{\pi}, \hat{\delta}, \hat{m} ; \tau, \xi, \eta)+\left(\frac{\partial \Phi}{\partial \pi}\right)_{\hat{\pi} \hat{\delta} \hat{m}} \Delta \pi+$

$+\left(\frac{\partial \Phi}{\partial \delta}\right)_{\hat{\hat{\pi}} \hat{\delta} \hat{m}} \Delta \delta+\left(\frac{\partial \Phi}{\partial m}\right)_{\hat{\hat{\pi}} \hat{\delta} \hat{m}} \Delta m+\frac{1}{2}\left(\frac{\partial^{2} \Phi}{\partial \pi^{2}}\right)_{\hat{\hat{\sigma}} \hat{\delta} \dot{m}}(\Delta \pi)^{2}+$

$+\frac{1}{2}\left(\frac{\partial^{2} \Phi}{\partial \delta^{2}}\right)_{\hat{\pi} \hat{\delta} \hat{m}}(\Delta \delta)^{2}+\frac{1}{2}\left(\frac{\partial^{2} \Phi}{\partial m^{2}}\right)_{\hat{\pi} \hat{\delta} \hat{m}}(\Delta m)^{2}+$

$+\left(\frac{\partial^{2} \Phi}{\partial \pi \partial \delta}\right)_{\hat{\tilde{x}} \hat{\delta} \hat{m}} \Delta \pi \Delta \delta+\left(\frac{\partial^{2} \Phi}{\partial \pi \partial m}\right)_{\hat{\pi} \hat{\delta} \hat{m} \dot{m}} \Delta \pi \Delta m+$

$+\left(\frac{\partial^{2} \Phi}{\partial m \partial \delta}\right)_{\hat{\pi} \hat{\delta} \hat{m}} \Delta m \Delta \delta$.

By substituting (23) into (18), representing $z(t)$ in the form (22) and neglecting the terms with the order of smallness greater than $\sigma_{i j}^{2}$ we get

$$
\begin{aligned}
& \hat{z}(t)=F(t)+ \\
& +\int_{0}^{t} \exp [-\hat{\pi}(t-\tau)] \int_{0}^{\tau} \int_{0}^{\xi} \hat{\Phi}(\tau, \xi, \eta) \hat{z}(\eta) d \eta d \xi d \tau, \quad \text { (24) } \\
& \sum_{i, j=1}^{3} \sigma_{i j}^{2} f_{i j}(t)= \\
& =\sum_{i, j=1}^{3} \sigma_{i j}^{2} \int_{0}^{t} \exp [-\hat{\pi}(t-\tau)] \int_{0}^{\tau} \int_{0}^{\xi} K_{i j} \hat{\Phi}(\tau, \xi, \eta) \hat{z} d \eta d \xi d \tau+ \\
& +\sum_{i, j=1}^{3} \sigma_{i j}^{2} \int_{0}^{t} \exp [-\hat{\pi}(t-\tau)] \int_{0}^{\xi} \int_{0}^{\xi} \hat{\Phi}(\tau, \xi, \eta) f_{i j}(\eta) d \eta d \xi d \tau,
\end{aligned}
$$

where

$$
\begin{aligned}
& \hat{\Phi}(\tau, \xi, \eta)=\hat{\pi} \hat{\delta} \hat{m} \exp [-(\hat{\delta} p+\hat{m} q)], p=\tau-\xi, \\
& q=\xi-\eta \text { and } K_{\pi \pi}=0, K_{\delta \delta}=p^{2} / 2-p / \hat{\delta}, \\
& K_{m m}=q^{2} / 2-q / \hat{m}, K_{\delta m}=\left(p-\hat{\delta}^{-1}\right)\left(q-\hat{m}^{-1}\right), \\
& K_{\pi \delta}=-\hat{\pi}^{-1}\left(p-\hat{\delta}^{-1}\right), K_{\pi n}=-\hat{\pi}^{-1}\left(q-\hat{m}^{-1}\right) .
\end{aligned}
$$


Equating the members at the same $\sigma_{i j}^{2}$ we get the equations for $f_{i j}$ : $f_{i j}(t)=F(t)_{i j}+$

$$
\begin{aligned}
& +\int_{0}^{t} \exp [-\hat{\pi}(t-\tau)] \int_{0}^{\tau} \int_{0}^{\xi} \hat{\Phi}(\tau, \xi, \eta) f_{i j}(\eta) d \eta d \xi d \tau, \\
& f_{i j}(0)=0
\end{aligned}
$$

where

$$
F_{i j}(t)=\int_{0}^{t} \exp [-\hat{\pi}(t-\tau)] \int_{0}^{\tau} \int_{0}^{\xi} K_{i j} \hat{\Phi}(\tau, \xi, \eta) z d \eta d \xi d \tau .
$$

The solution of (27) is equivalent to the solution of (15) for homogeneous vegetation cover, when $\pi(\omega)=\hat{\pi}, \delta(\omega)=\hat{\delta}$ and $m(\omega)=\hat{m}$. Then we get from (15) the following equation for $\hat{z}(t)$ :

$$
\begin{aligned}
& \frac{d^{2} \hat{z}}{d t^{2}}+a \frac{d \hat{z}}{d t}+b \hat{z}= \\
& =\frac{1}{C^{*}}\left[\frac{d Q}{d t}+(\hat{m}+\hat{\delta}) Q+\hat{m} \hat{\delta} \int_{0}^{t} Q d \tau\right],
\end{aligned}
$$

where $a=\hat{\pi}+\hat{m}+\hat{\delta}, b=\hat{\pi} \hat{m}+\hat{\pi} \hat{\delta}+\hat{m} \hat{\delta}$ with the initial conditions: $\hat{z}(0)=0, d \hat{z} / d t(0)=Q(0) / C^{*}$. The problem has the following solution:

$$
\begin{aligned}
& \hat{z}(t)=\frac{\hat{m} \hat{\delta}}{b} \int_{0}^{t} \frac{Q(\tau)}{C^{*}} d \tau+\frac{1}{C^{*} \sqrt{a^{2}-4 b}} \cdot \\
& \cdot\left\{\left(\hat{m}+\hat{\delta}+\lambda_{1}+\frac{\hat{m} \hat{\delta}}{\lambda_{1}}\right) \int_{0}^{t} Q(\tau) \exp \left[\lambda_{1}(t-\tau)\right] d \tau-\right. \\
& \left.-\left(\hat{m}+\hat{\delta}+\lambda_{2}+\frac{\hat{m} \hat{\delta}}{\lambda_{2}}\right) \int_{0}^{t} Q(\tau) \exp \left[\lambda_{2}(t-\tau)\right] d \tau\right\},
\end{aligned}
$$

where

$$
\lambda_{1}=\frac{1}{2}\left(-a+\sqrt{a^{2}-4 b}\right), \lambda_{2}=-\frac{1}{2}\left(a+\sqrt{a^{2}-4 b}\right) .
$$

But at the same time this solution is also the solution of equation (24). On the other hand, equation (18) coincides with (24) if the function $F(t)$ is replaced by the function

$$
F_{i j}(t)=\int_{0}^{t} \exp [-\hat{\pi}(t-\tau)] \int_{0}^{\tau} \int_{0}^{\xi} K_{i j} \hat{\Phi}(\tau, \xi, \eta) \hat{z} d \eta d \xi d \tau .
$$

Therefore the solution of (27), $f_{i j}(t)$, can be presented in the form of (29) with the modified function of emission

$$
Q(\tau) \Rightarrow Q_{i j}(\tau)=\int_{0}^{\tau} \int_{0}^{\xi} K_{i j} \hat{\Phi}(\tau, \xi, \eta) z d \eta d \xi d \tau
$$

\section{REFERENCES}

Bazilevich, N.I., Biogeochemistry of the Earth and functional models of exchange processes in natural ecosystems, Modern problems of biogeochemistry, Moscow, Nauka: 56$73,1979$.
Bazilevich, N.I., Biological Productivity of Ecosystems of Northern Eurasia, Moscow, Nauka, 293 pp., 1993.

Bazilevich, N.I. and Rodin, L.E., Geographical regularities of productivity and turnover of chemical elements for the main types of Earth's vegetation, General theoretical problems of biological productivity, Leningrad, Nauka: 24-32, 1969.

Bazilevich, N.I., Grebentshikov, O.S. and Tishkov, A.A., Geographical regularities of the structure and functioning of ecosystems, Moscow, Nauka, 297pp., 1986.

Cherkinsky, A. and Brovkin, V., A model of humus formation in soils based on radiocarbon data of natural ecosystems, Radiocarbon, 33(2), 186-187, 1991.

den Elzen, M., Beusen, A. and Rotmans, J., Modelling Global Biogeochemical Cycles: an integrated assessment approach, GLOBO Report Series no. 7, RIVM report no. 462502007, 104pp., 1995.

Foley, J.A., Prentice, I.C., Ramankutty, N., Levis, S., Pollard, D., Sitch, S. and Haxeltine, A., An integrated biosphere model of land surface processes, terrestrial carbon balance, and vegetation dynamics, Global Biogeochemical Cycles, 10(4): 603-628, 1996.

Friend, A.D., Stevens, A.K., Knox, R.G., Cannel, M.G.R., A process based terrestrial biosphere model of ecosystem dynamics (Hybrid v3.0), Ecological Modelling, 95, 249-288, 1997.

Haxeltine, A., Prentice, I.C., BIOME3: An equilibrium terrestrial biosphere model based on ecophysiological constraints, resource availability, and competition among plant functional types, Global Biogeochemical Cycles, 10(4), 693-710, 1996.

Kwon, O. and Schnoor, J.L., Simple global carbon model: the atmosphere - terrestrial biosphere - ocean interaction, Global Biogeochemical Cycles, v. 8, 3, 295-305, 1994.

Svirezhev, Yu., Brovkin, V., von Bloh, W., Schellnhuber, H.-J. and Petschel-Held, G., Optimisation of reduction of global $\mathrm{CO}_{2}$ emission based on a simple model of the carbon cycle, Environmental Modeling and Assessment, 4, 23-33, 1999.

Svirezhev, Yu., Krapivin, V.F. and Tarko, A.M., Modeling of the main biosphere cycles, Global Change, T.F. Malone and J.S. Roederer (Eds), Cambridge Univ. Press, Cambridge, 298-313, 1985. 